\title{
A conservative therapy for patient with unicystic ameloblastoma and impacted premolar using decompression combined with orthodontic treatment-a case report
}

\author{
Yabing Dong ${ }^{1,2,3,4,5}$, Jingang Yang ${ }^{1,3,4,5}$, Quan Yu ${ }^{3,4,5,6}$, Shanghui Zhou ${ }^{1,3,4,5}$ \\ ${ }^{1}$ Department of Oral \& Maxillofacial - Head \& Neck Oncology, Shanghai Ninth People's Hospital, Shanghai Jiao Tong University School of \\ Medicine, Shanghai, China; ${ }^{2}$ Department of Oral Surgery, Shanghai Ninth People's Hospital, Shanghai Jiao Tong University School of Medicine, \\ Shanghai, China; ${ }^{3}$ College of Stomatology, Shanghai Jiao Tong University, National Center for Stomatology, Shanghai, China; ${ }^{4}$ National Clinical \\ Research Center for Oral Diseases, Shanghai, China; ${ }^{5}$ Shanghai Key Laboratory of Stomatology, Shanghai, China; ${ }^{6}$ Department of Orthodontics, \\ Shanghai Ninth People's Hospital, Shanghai Jiao Tong University School of Medicine, Shanghai, China \\ Correspondence to: Quan Yu, PhD. Department of Orthodontics, Shanghai Ninth People's Hospital, Shanghai Jiao Tong University School of \\ Medicine, 639 Zhi-Zao-Ju Road, Shanghai 200011, China. Email: quanyu122@vip.sina.com; Shanghui Zhou, PhD, Department of Oral \& \\ Maxillofacial - Head \& Neck Oncology, Shanghai Ninth People's Hospital, Shanghai Jiao Tong University School of Medicine, 639 Zhi-Zao-Ju \\ Road, Shanghai 200011, China. Email: zhoushanghui@shsmu.edu.cn.
}

Background: Ameloblastoma may cause a series of complications, such as facial deformity and functional disability. It's a dilemma whether to treat adolescent ameloblastoma patients with initial radical treatment, extensive surgery or conservative procedures. The radical treatment could result in deformity and dysfunction of the jaw that are bound to influence both the physical and psychological development of the patient in later life. And the surgical excision of the surrounding tissues and the extraction of affected teeth could result in the defects in the dentition function and aesthetics, which make the reconstruction of the occlusion remains challenging.

Case Description: We describe the successful interdisciplinary orthodontic treatment combined with decompression of a patient with ameloblastoma. A 10-year-old female with a UAM in the mandible and the right mandibular second premolar impacted was recruited. The patient received the comprehensive orthodontic treatment combined with the decompression of the cystic lesions. Panoramic radiographs indicated that the dentoalveolar bone of the UAM region was well healed. The impacted right mandibular second premolar erupted eventfully, with no evidence of recurrence in 34 months. The malocclusion was corrected and appeared stable after the orthodontic treatment.

Conclusions: Our findings in this case report suggest that the combination of conservative therapy and orthodontic treatment is one of an effective management option to reconstruct the occlusion of an adolescent patient with a UAM in the mandible. The application of decompression eliminated the UAM, which may promote the formation of new bone in the ameloblastoma region. Considering the risk of recurrence of UAM, the postoperative radiographic and clinical follow-up is necessary.

Keywords: Ameloblastoma; decompression; impacted premolar; orthodontic treatment; case report

Received: 07 March 2021; Accepted: 08 June 2021; Published: 10 September 2021.

doi: 10.21037/fomm-21-17

View this article at: https://dx.doi.org/10.21037/fomm-21-17

\section{Introduction}

The unicystic ameloblastoma (UAM) is one of the most common benign odontogenic tumors of epithelial origin, with strong local invasiveness and involving the mandible more frequently than the maxilla. With the extension of the course of the UAM, the cyst volume gradually increases and implicates multiple teeth. Meanwhile, due to the increasing pressure of the cyst cavity, the teeth affected by the UAM are 
usually unable to erupt normally. The common treatment of cystic lesions containing teeth is a surgical therapy (1). Regarding the treatment of UAM, surgical excision of surrounding tissues is still the general approach. However, the extraction of affected teeth during the operation would result in the defects in the dentition function and aesthetics. With advantages of simple operation, small surgical trauma, a low postoperative recurrence rate and maintaining the structure and the function of the surrounding tissue, the decompression technique is proved to be an effective treatment of the cystic lesions (2). Several clinical studies reported that the jaw cystic lesions decompression surgery combined with orthodontic treatment might help preserve the permanent teeth and correct the malocclusion caused by ameloblastoma $(3,4)$. In this case report, an adolescent patient diagnosed with a UAM in the mandible and the mandibular second premolar impacted was treated by the comprehensive orthodontic therapy and the decompression of the cystic lesions. The timing and role of the orthodontic interventions in patients with mandibular ameloblastoma was also discussed.

Ameloblastoma is an aggressive odontogenic tumor that originates from odontogenic epithelium within a mature fibrous stroma devoid of odontogenic ectomesenchyme (5). In order of frequency, the benign ameloblastoma include (I) solid or multicystic ameloblastoma is the most common type and radiologically characterized by multilocular aspect, (II) unicystic ameloblastoma, radiologically characterized by unilocular aspect, (III) peripheral or extraosseous ameloblastoma, and (IV) desmoplastic ameloblastoma. UAMs account for about $5-15 \%$ of all ameloblastoma cases frequently detected in younger patients with average age of 26.1 years (6). Ameloblastoma usually occur in the posterior mandible manifested as an asymptomatic swelling (7). The majority of UAMs resemble dentigerous cysts because of their association with an unerupted tooth. The UAM is thought to have recurrence potential, but to be less aggressive than the solid type.

\section{Case presentation}

All procedures performed in studies involving human participants were in accordance with the ethical standards of the institutional and national research committee and with the Helsinki Declaration (as revised in 2013). This research was approved by the Ethics Review Committee of Shanghai Ninth People's Hospital affiliated to Shanghai Jiao Tong University, School of Medicine (NO.: SH9H-
2021-T148-1). Photographs and participation in the study were authorized by the patient's parents. Informed consent was obtained from the participant with a detailed description of the purpose and benefits from the study.

\section{Diagnosis and etiology}

A 10 years and 2 months old female with a UAM in the mandible and the mandibular second premolar impacted was referred to the Department of Oral \& Maxillofacial Head \& Neck Oncology, Shanghai Ninth People's Hospital, Shanghai Jiao Tong University School of Medicine. The panoramic radiographs and dental casts were obtained (Figure 1). Radiologically, the right mandibular second premolar was suggested impacted, which was completely surrounded by a radiolucency with a well-defined corticated border that looked like a cystic lesion. The neighboring teeth were affected. The swelling extended to the right mandibular first molar both buccally and lingually, and it felt soft in palpation. The clinical and radiographic findings, suggested a dentigerous cyst primary.

The patient underwent the orthodontic operation at 11 years and 5 months old. The extraoral examination revealed mild facial asymmetry with the chin deviated to the right side. The intraoral examination, showed Class I malocclusion of the patient, with a mild crowding in both arches. The upper midline of the arch deviated to the right side slightly (Figure 2). Panoramic radiographs demonstrated no pathology. Cephalometric analysis revealed a Class II skeletal relationship and a hyperdivergent growth pattern. The lower incisor inclination was reduced.

\section{Treatment objectives}

The problem list for this patient included the presence of a Class I malocclusion characterized by a condition of mild crowding in the posterior region of mandibular arches, a labially positioned right mandibular second premolar, a mild deviation of the maxillary dental arch midline. A comprehensive orthodontic treatment aiming to correct the above-mentioned genetic and iatrogenic malocclusion was proposed and accepted by the patient.

\section{Treatment progress}

In consultation with the Department of Orthodontics, an attempted was made to allow the impacted tooth to erupt with the decompression technique. In the Department of 

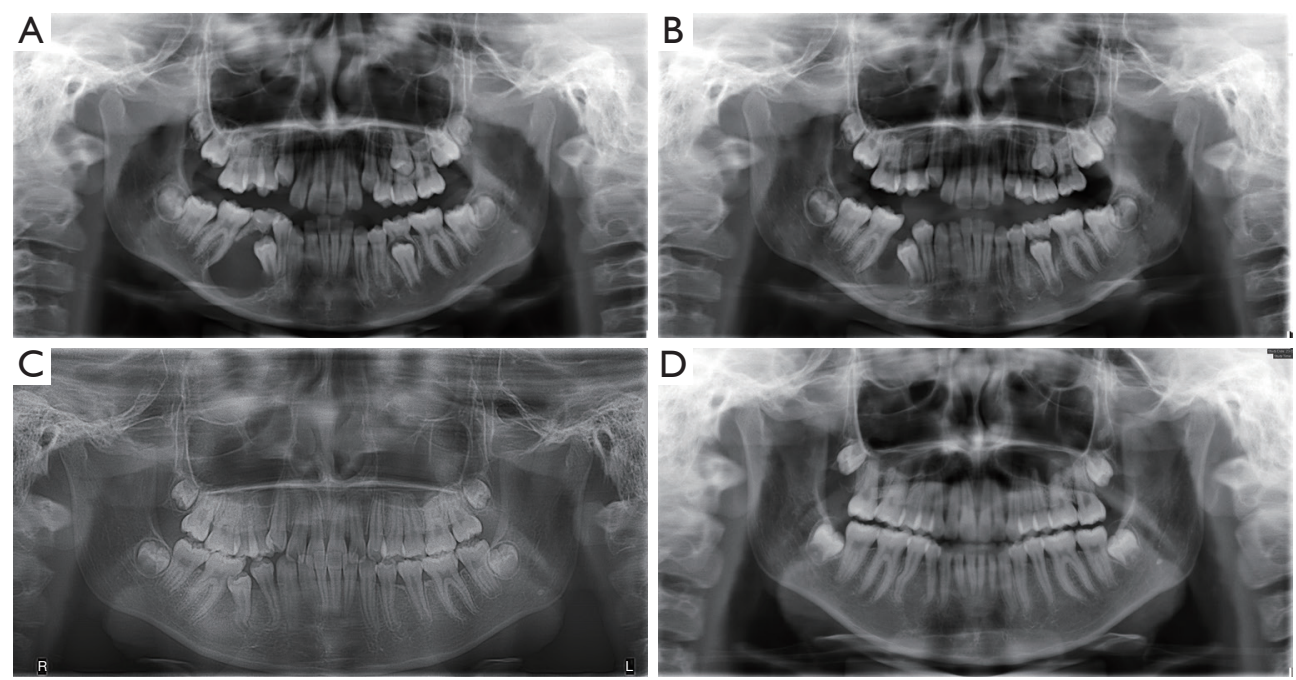

Figure 1 Panoramic radiographs, (A) before the decompression, (B) 3 months post-decompression, (C) before the orthodontic treatment, (D) after the orthodontic treatment.
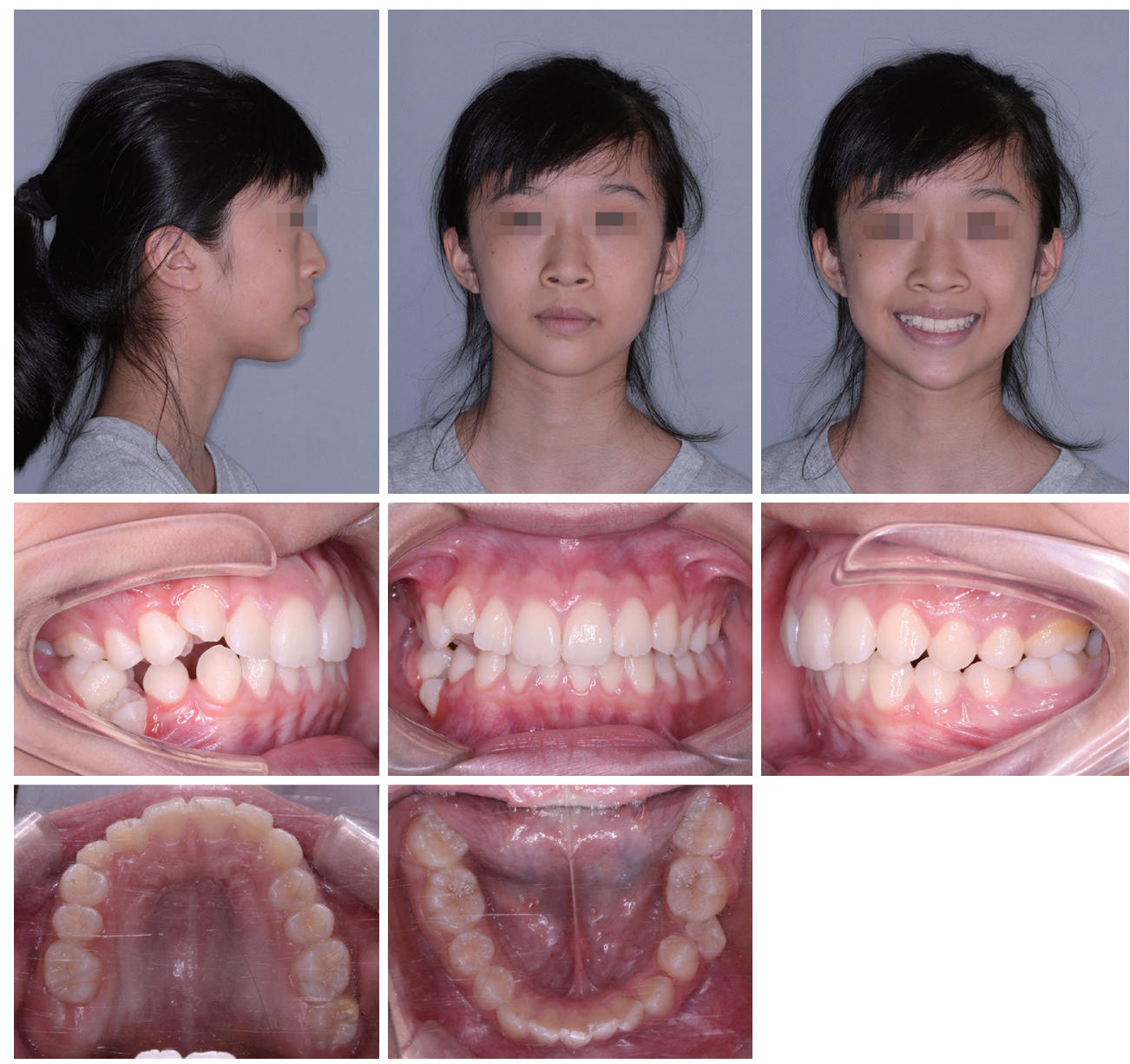

Figure 2 Facial and intraoral photographs when the orthodontic treatment initiated. 
Oral \& Maxillofacial - Head \& Neck Oncology, instead of to remove the right mandibular cystic lesions (Figure 3). The patient wore an obturator designed for a cavity using the impression model for 8 months. The patient's biopsy sample was histologically classified as the UAM (Figure 4). After the lesion was reduced, a complete curettage was performed, and the right mandibular second premolar was fenestrated to facilitate its eruption. The operation was successfully completed and the recovery was good (Figure 5).

When the orthodontic treatment initiated the maxillary and mandibular dental arch was bonded 0.022-inch slots preadjusted edgewise appliance in order to level and align the dental arch. The patient's maxillary and mandibular arch was leveled with continuous archwires, starting with 0.014 -in nickel titanium and working up to $0.019^{*} 0.025$-in stainless steel to control torque. During the finishing stage, after the molars were in Class I occlusion, final detailing of the occlusion was accomplished with $0.019^{*} 0.025$-in stainless steel archwires in conjunction with triangle elastics from the maxillary canines to the mandibular canines and second premolars to settle the occlusion and establish an ideal occlusion (Figure 6). Fixed retainers were attached to the maxillary and mandibular anterior teeth. Wraparound removable retainers were also delivered to secure the stability of both arches (Figure 7). The orthodontic treatment lasted 22 months, during which there were no indications of ameloblastoma recurrence.

\section{Treatment results}

After 22-month orthodontic treatment with fixed appliances, acceptable overbite and overjet as well as Class I canine and molar relationships were achieved. A posttreatment panoramic radiograph showed satisfactory root parallelism of incisors and no significant alveolar bone defects in the right mandibular second premolar and first molar (Figure 8). Superimposition of the initial and final tracings of the lateral cephalometric $\mathrm{X}$-rays indicated a slight labial proclination of both the maxillary and the mandibular incisors occurred after treatment (U1-SN, 104.6 ${ }^{\circ}$ IMPA, $97.5^{\circ}$, Figure 8 , Table 1). The patient wore the retainer to maintain the stability of the occlusion relationship. The CBCT images showed stable treatment results after 34-month follow-up and no significant ameloblastoma recurrence.

\section{Discussion}

Whether to treat adolescent UAM patients with initial radical operation is always a difficult decision to make $(1,8,9)$. Enucleation, curettage and decompression are available surgical treatment options, which can result in deformity and dysfunction of the mandible, thereby impeding both the physical and psychological development of the patient in later life (10). Enucleation and curettage are commonly applied to the treatment of an adenomatoid odontogenic tumor, but they usually lead to tooth loss (11). Preoperative confirmation of the histological type by means of incisional biopsy would contribute to the selection of appropriate treatment modalities, which can possibly reduce the recurrence rate. Several methods can be used in oral rehabilitation, including prosthodontics, auto-transplantation and dental implants, which can be preceded by bone grafts or osteogenesis distraction (12-14). However, patients are not always candidates for these procedures because of age-related or financial reasons. Autogenous tooth transplantation cost less than dental implants, but its application was limited by the secondary injury and the lower success rate. There is a trend in the scientific community in recent years for less invasive therapeutic procedures for ameloblastomas, considering the clinical, radiographic, and histopathologic variables. Several literatures about the combination of conservative surgery and orthodontic treatment indicated it an effective management options for UAM in recent years $(4,5)$. Compared with enucleation and curettage surgeries, decompression decreases the size of the cyst, reduces the extent of the bone defect, promotes the eruption of a cyst-associated tooth, and helps prevent the wide resection of the mandible in patients with UAM (15). The decompression achieves the bone reconstruction effects by balancing the internal and external pressure of the tumor, eliminating or reducing the bone resorption factors of the cyst, and changing the lining epithelium of the cyst. UAM disappeared without a second-stage curettage in this case. The change of environment after decompression may inhibit the growth of cyst epithelium, and the further research is needed to explain this situation. Moreover, decompression is capable of preserving important anatomical structures, such as inferior alveolar nerves, preventing consequent possible deformity and improvements of patients' quality of life post-surgery. In comparison with the extraction of the impacted tooth, the decompression technique provides an opportunity for tooth eruption, and as a result, it's possible to preserve both the jaw and teeth. It is worth noting that simple decompression is not enough to eradicate the lesion completely. The second-stage curettage to completely 

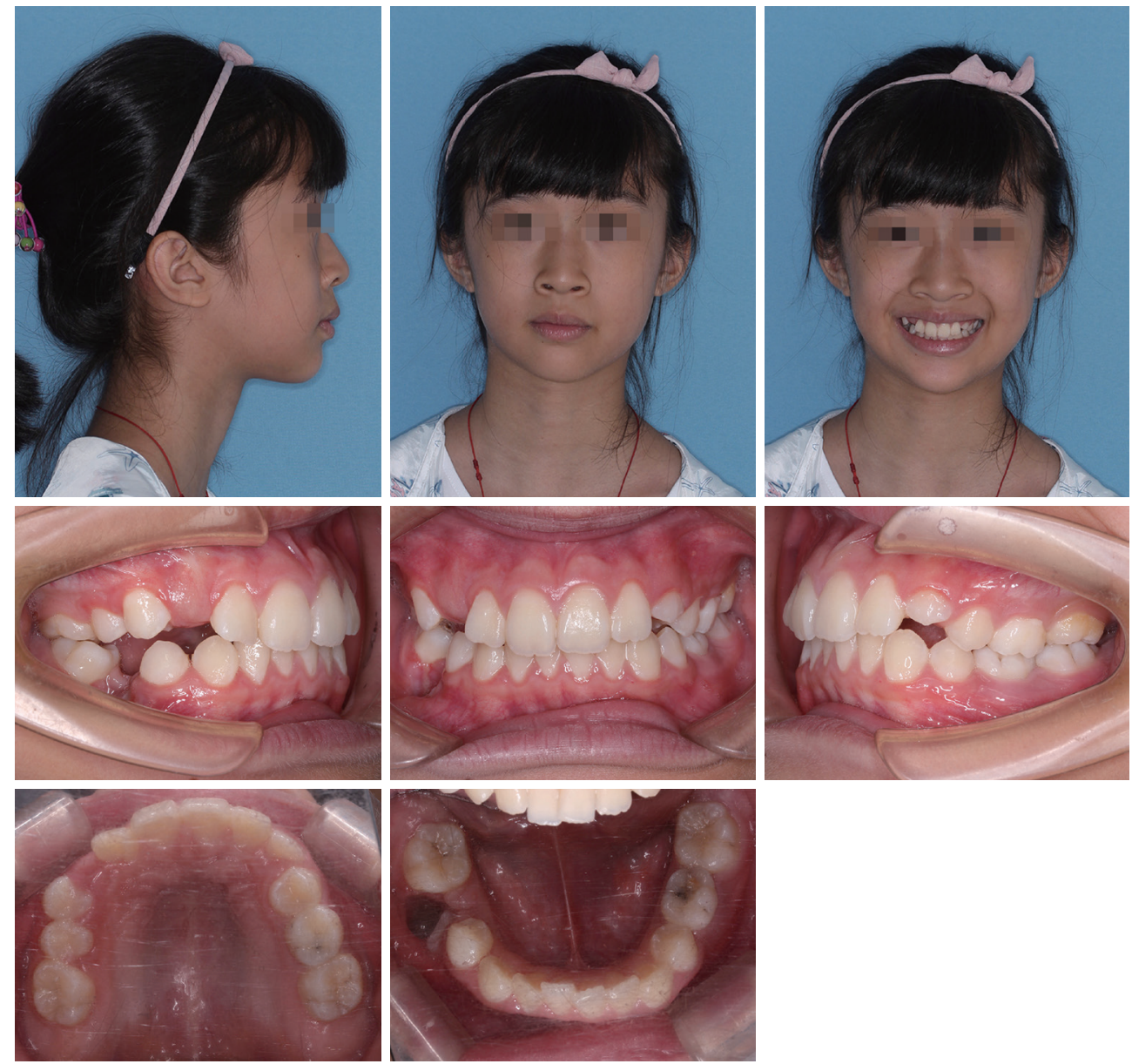

Figure 3 Facial and intraoral photographs after the decompression.
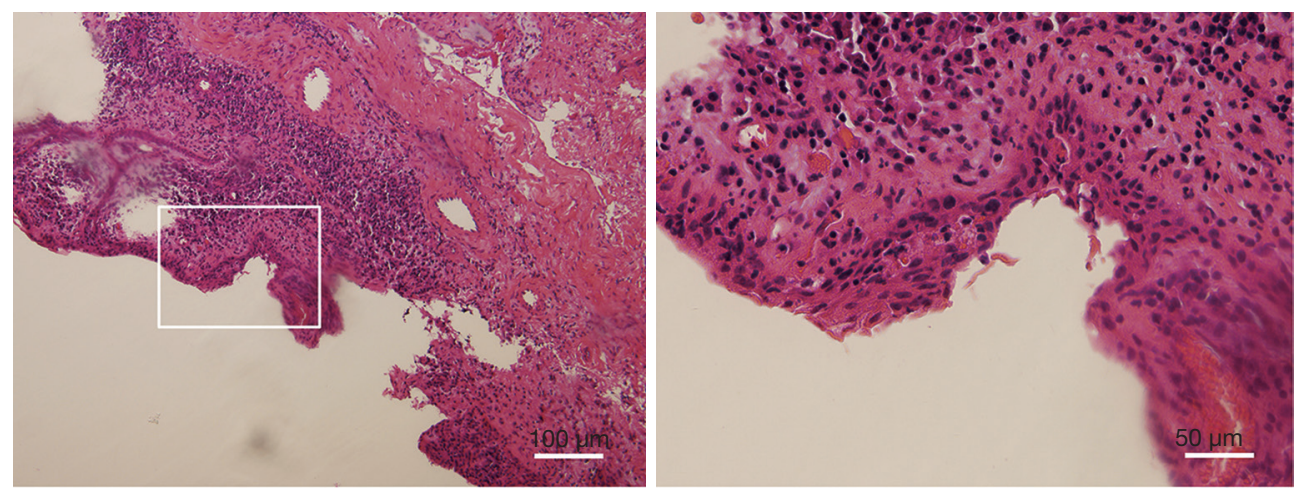

Figure 4 The histological findings of the specimen obtained at the cystic wall tissue of the unerupted right mandibular second premolar stained with Hematoxylin Eosin. 

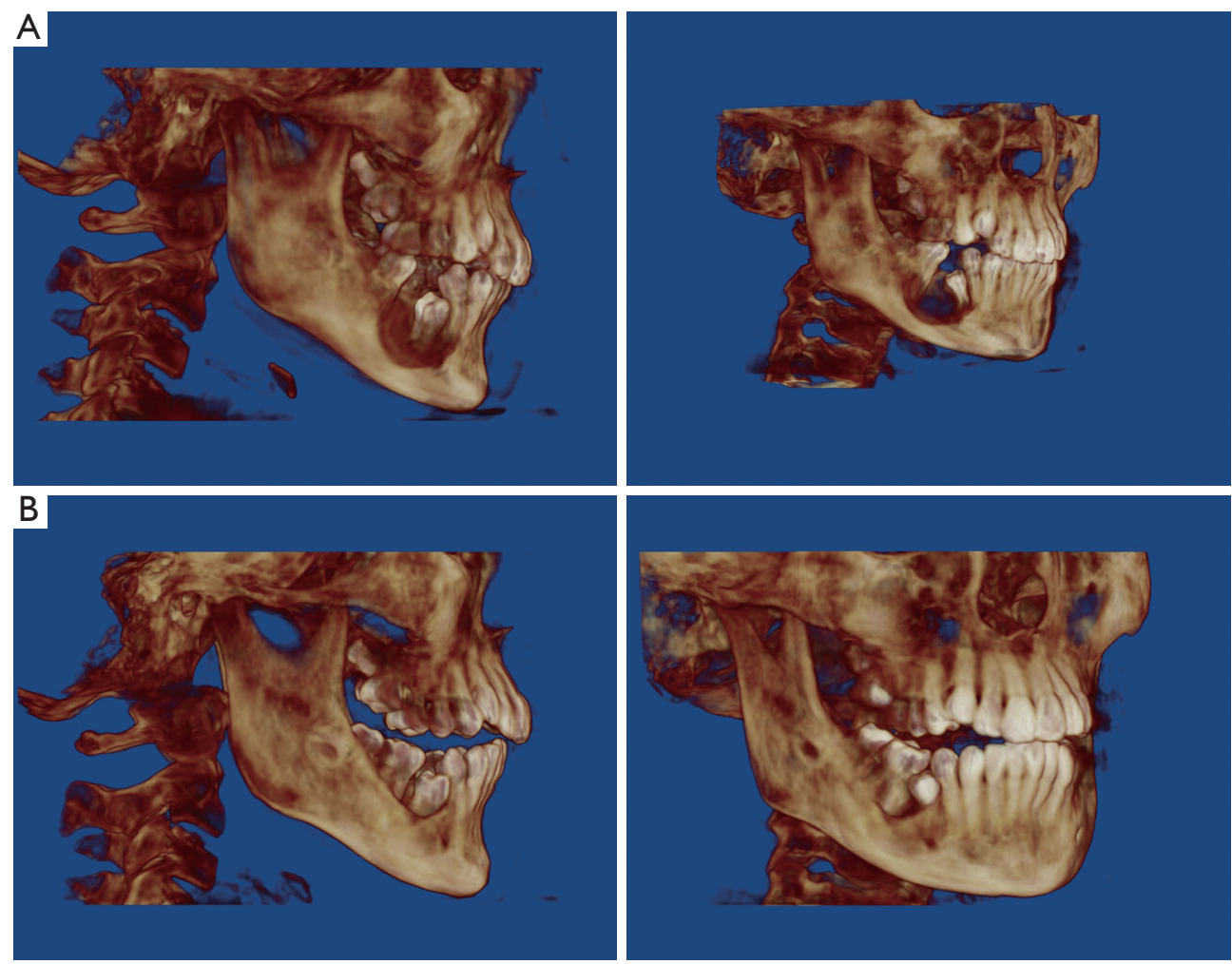

Figure 5 Cone-Beam computed tomography images was performed (A) before the decompression, (B) after the impacted right mandibular second premolar eruption, the orthodontic treatment initiated.
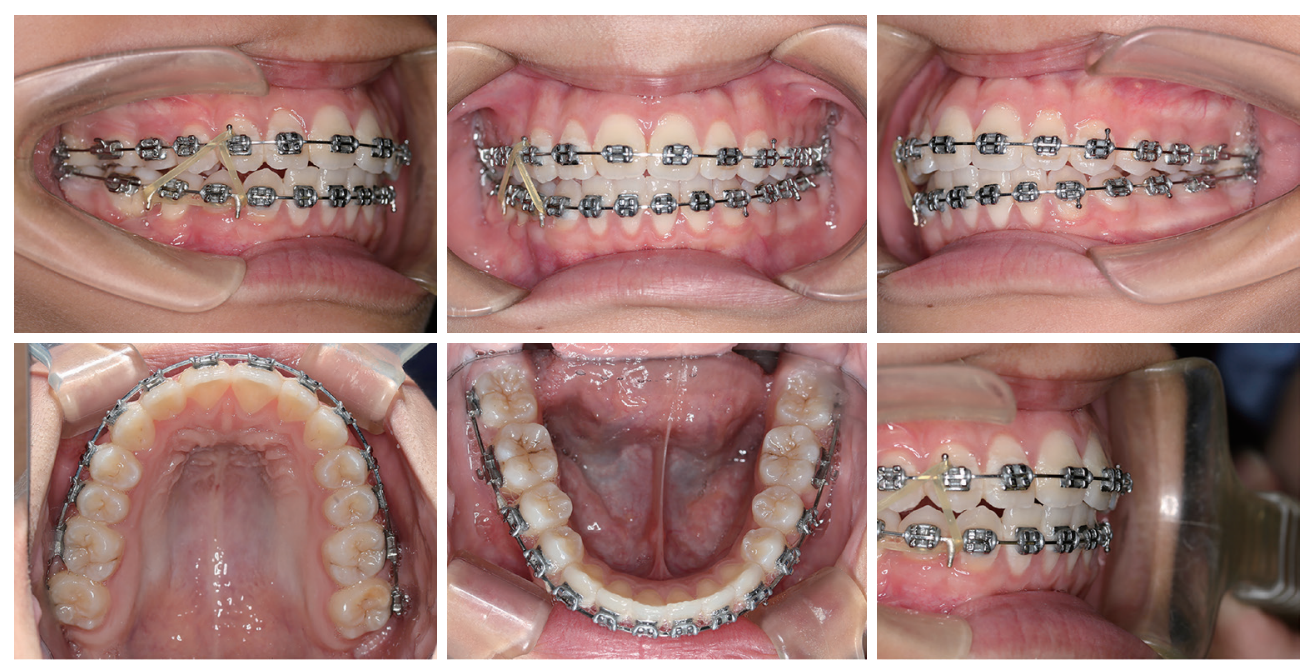

Figure 6 Triangle elastics in the right posterior teeth to settle the occlusion and establish an ideal occlusion.

remove the cystic lesion is still necessary for most patients.

The present case report represents a case with a UAM in the mandible treated by the interdisciplinary orthodontic therapy and decompression of the cystic lesions. A prosthetic approach was also considered as an alternative, but it was a compromised process because it might affect the normal eruption of the impacted premolar. After decompression, the appropriate timing of the orthodontic 

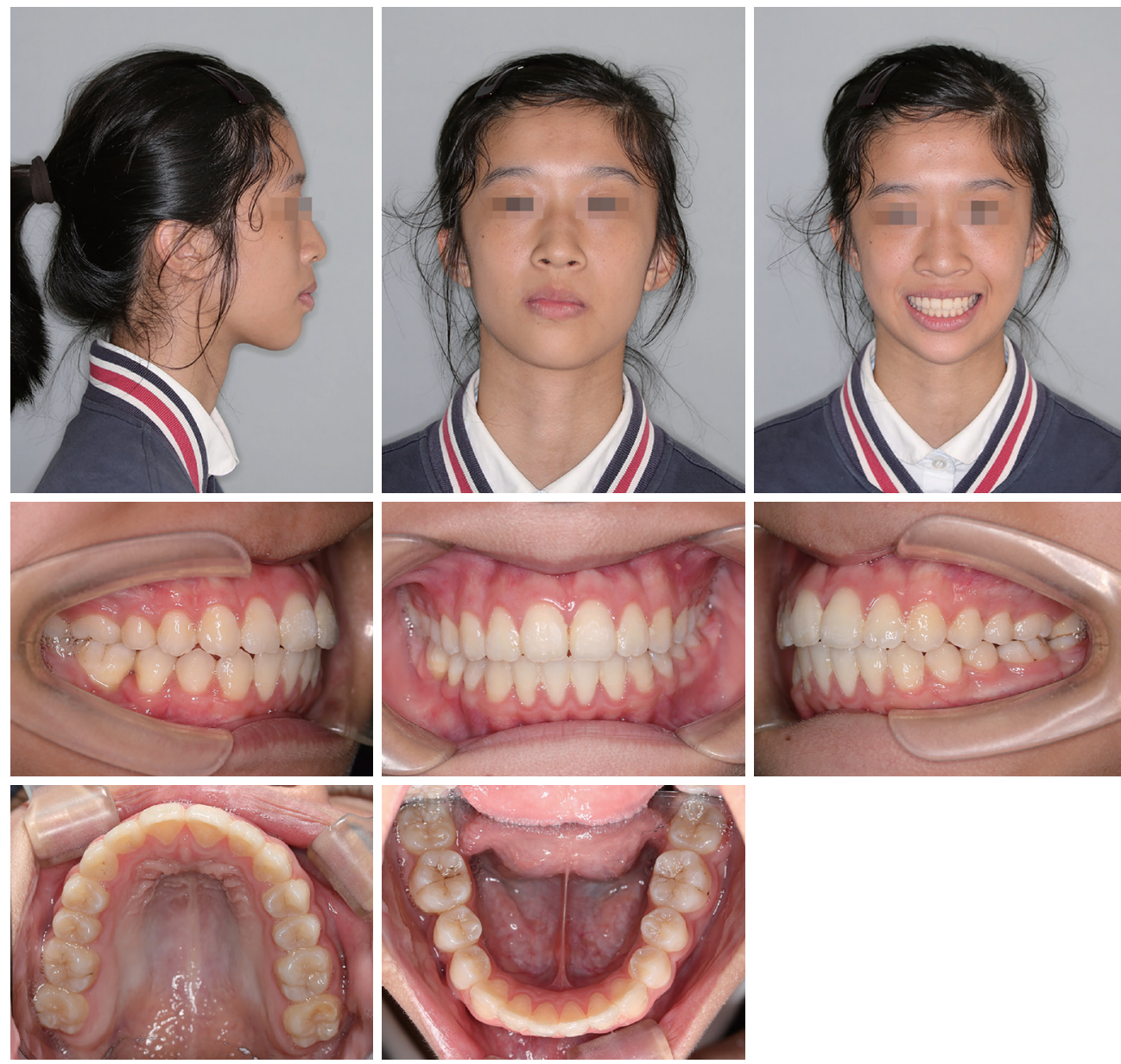

Figure 7 Posttreatment extraoral and intraoral photographs. The patient had an attractive smile, normal overjet and overbite, Class I canine and molar relationships, and coincident dental midline.
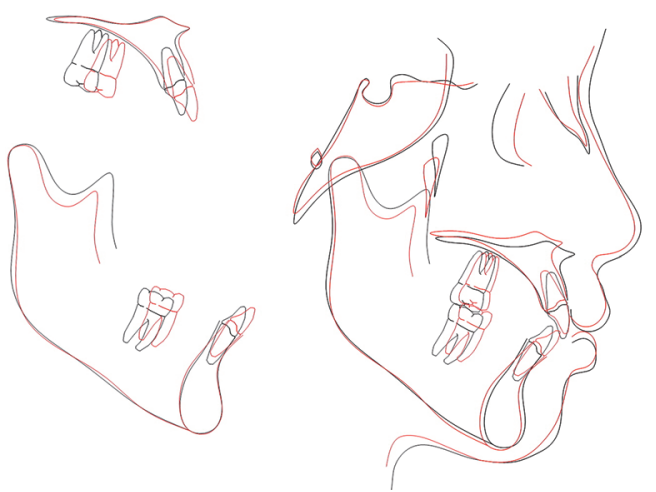

Figure 8 Superimposition of lateral cephalometric tracings on the palatal vault and zygomas, on the inner surface of the mandibular symphysis and outline of the mandibular canal, and on the sellanasion plane at sella. Black, pretreatment; red, posttreatment. intervention is important. Because the roots of the affected teeth have not yet developed and the bone wall surrounding the tumor has not been reconstructed. Therefore, panoramic pictures and CBCT should be taken regularly. Sometimes in order to understand the changes of the tumor, it is necessary to supplement with enhanced CT. If the affected tooth has no effect on the roots of adjacent teeth in the subsequent treatment process, the affected tooth would be well supported, which is conductive to the formation of the roots of the affected tooth. The orthodontic traction at this time can achieve a good therapeutic effect. The decompression can promote the formation of new bone in the ameloblastoma region. The eruption and movement of the right mandibular second premolar was observed during 
Table 1 Cephalometric measurements

\begin{tabular}{lccc}
\hline Measurement & Norm & Pretreatment & Posttreatment \\
\hline SNA $\left(^{\circ}\right)$ & 82.0 & 79.9 & 80.1 \\
SNB $\left(^{\circ}\right)$ & 77.7 & 75.3 & 76.4 \\
ANB $\left(^{\circ}\right)$ & 4.0 & 4.6 & 3.6 \\
Wits $(m m)$ & -1.0 & 1.7 & -1.7 \\
SN-MP $\left(^{\circ}\right)$ & 33.0 & 41.8 & 43.3 \\
Ramus height $(m m)$ & 41.0 & 38.6 & 39.8 \\
LFH:TFH & 55.0 & 52.3 & 55.6 \\
(ANS-Me/N-Me) $(\%)$ & & & \\
U1-SN $\left(^{\circ}\right)$ & 103.4 & 96.1 & 104.6 \\
U1-NA $\left(^{\circ}\right)$ & 22.8 & 16.1 & 24.6 \\
U1-NA (mm) & 4.0 & 3.0 & 6.4 \\
IMPA $\left(^{\circ}\right)$ & 96.8 & 87.6 & 97.5 \\
L1-NB $\left(^{\circ}\right)$ & 25.0 & 24.7 & 37.3 \\
L1-NB (mm) & 4.0 & 5.3 & 9.2 \\
U1/L1 $\left(^{\circ}\right)$ & 124.0 & 134.5 & 114.5 \\
Upper lip to E-plane $(\mathrm{mm})$ & 1.0 & 0.8 & -1.2 \\
Lower lip to E-plane $(\mathrm{mm})$ & 2.0 & 2.3 & 3.9 \\
\hline
\end{tabular}

the orthodontic treatment. The acceptable Class I canine and molar relationships were achieved and the alveolar bone defects in the right mandibular second premolar and first molar was healed. The whole course of orthodontic treatment was acceptable. The short-term positive effects demonstrated that the interdisciplinary approach could well restore both the occlusal function and normal facial appearance. There were a few limitations to the current case report-The case report was retrospective. Considering the risk of recurrence of UAM, the postoperative follow-up is necessary. Radiographic and clinical follow-up should be done every 6 months for the first 2 years and then annually.

\section{Conclusions}

The present case report demonstrates that the decompression technique may inhibit the growth and progression of the UAM. The decompression technique also promotes the bone formation and tooth movement in the ameloblastoma region, which enables the eruption of impacted premolar. The multidisciplinary approach including orthodontic treatment helps to reconstruct a normal and stable occlusal function.

\section{Acknowledgments}

Funding: This project was supported by the grants of Shanghai Pujiang Program (No. 18PJD026)

\section{Footnote}

Peer Review File: Available at https://fomm.amegroups.com/ article/view/10.21037/fomm-21-17/prf

Conflicts of Interest: All authors have completed the ICMJE uniform disclosure form (available at https://fomm. amegroups.com/article/view/10.21037/fomm-21-17/coif). The authors have no conflicts of interest to declare.

Ethical Statement: The authors are accountable for all aspects of the work in ensuring that questions related to the accuracy or integrity of any part of the work are appropriately investigated and resolved. All procedures performed in studies involving human participants were in accordance with the ethical standards of the institutional and/or national research committee(s) and with the Helsinki Declaration (as revised in 2013). This research was approved by the Ethics Review Committee of Shanghai Ninth People's Hospital affiliated to Shanghai Jiao Tong University, School of Medicine (NO.: SH9H-2021-T148-1). Photographs and participation in the study were authorized by the patient's parents. Informed consent was obtained from the participant with a detailed description of the purpose and benefits from the study.

Open Access Statement: This is an Open Access article distributed in accordance with the Creative Commons Attribution-NonCommercial-NoDerivs 4.0 International License (CC BY-NC-ND 4.0), which permits the noncommercial replication and distribution of the article with the strict proviso that no changes or edits are made and the original work is properly cited (including links to both the formal publication through the relevant DOI and the license). See: https://creativecommons.org/licenses/by-nc-nd/4.0/.

\section{References}

1. Effiom OA, Ogundana OM, Akinshipo AO, et al. Ameloblastoma: current etiopathological concepts and management. Oral Dis 2018;24:307-16. 
2. Morankar R, Bhatia SK, Goyal A, et al. Conservative management of keratocystic odontogenic tumour in a young child with decompression and an intraoral appliance: 5-year follow-up. BMJ Case Rep 2018;2018:bcr2017221563.

3. Morice A, Neiva C, Fabre M, et al. Conservative management is effective in unicystic ameloblastoma occurring from the neonatal period: A case report and a literature review. Oral Surg Oral Med Oral Pathol Oral Radiol 2020;129:e234-42.

4. Takahashi S, Idaira Y, Sato T, et al. Unicystic ameloblastoma in a child treated with a combination of conservative surgery and rrthodontic rreatment: a case report. J Clin Pediatr Dent 2019;43:121-5.

5. Speight PM, Takata T. New tumour entities in the 4th edition of the World Health Organization Classification of Head and Neck tumours: odontogenic and maxillofacial bone tumours. Virchows Arch 2018;472:331-9.

6. Dhanuthai K, Chantarangsu S, Rojanawatsirivej S, et al. Ameloblastoma: a multicentric study. Oral Surg Oral Med Oral Pathol Oral Radiol 2012;113:782-8.

7. Bansal S, Desai RS, Shirsat P, et al. The occurrence and pattern of ameloblastoma in children and adolescents: an Indian institutional study of 41 years and review of the literature. Int J Oral Maxillofac Surg 2015;44:725-31.

8. Ghandhi D, Ayoub AF, Pogrel MA, et al. Ameloblastoma: a surgeon's dilemma. J Oral Maxillofac Surg

doi: $10.21037 /$ fomm-21-17

Cite this article as: Dong Y, Yang J, Yu Q, Zhou S. A conservative therapy for patient with unicystic ameloblastoma and impacted premolar using decompression combined with orthodontic treatment-a case report. Front Oral Maxillofac Med 2021;3:30.
2006;64:1010-4.

9. Carlson ER, Marx RE. The ameloblastoma: primary, curative surgical management. J Oral Maxillofac Surg 2006;64:484-94.

10. Xu L, Li C, Wang H, et al. Distraction osteogenesis of fibula graft for mandibular reconstruction following ameloblastoma ablation. J Craniofac Surg 2019;30:202-4.

11. Ishihara $\mathrm{Y}$, Arakawa H, Nishiyama A, et al. Occlusal reconstruction of a patient with ameloblastoma ablation using alveolar distraction osteogenesis: a case report. Head Face Med 2020;16:12.

12. Osterne RL, Moreira Neto JJ, de Araújo Lima AD, et al. Autotransplantation of immature third molars and orthodontic treatment after en bloc resection of conventional ameloblastoma. J Oral Maxillofac Surg 2015;73:1686-94.

13. Block MS. Dental Implants: The Last 100 Years. J Oral Maxillofac Surg 2018;76:11-26.

14. Bianchi AE, Vinci R, Torti S, et al. Atrophic mandible reconstruction using calvarial bone grafts and implantsupported overdentures: radiographic assessment of autograft healing and adaptation. Int J Periodontics Restorative Dent 2004;24:334-43.

15. Castro-Núñez J. Decompression of Odontogenic Cystic Lesions: Past, Present, and Future. J Oral Maxillofac Surg 2016;74:104.e1-9. 